\title{
The perception of multiple simultaneously presented forms as a function of foveal spacing '
}

Using five capital letters as the form stimuli and tachistoscopic presentation, an exposure duration was determined for each $S$ that yielded $80 \%$ identification accuracy when single letter displays were presented. Then the increment in exposure duration necessary for a correct identification of all letters on a display on $80 \%$ of the trials was determined for 2, 3, and 4 letter displays. In view of evidence that perceptual independence breaks down when stimuli are spaced much closer than $1^{\circ}$ apart in the fovea, the effect of different foveal spacing of the form stimuli in the display was studied. Spacings of $1 / 2,3 / 4$, and $1^{\circ}$ of angle were employed. Less than a $30 \%$ increment in exposure duration was necessary to recognize 2 form displays at the same accuracy level as single form. But no further increase in exposure duration was necessary to recognize 3 and 4 form displays at the same accuracy criterion. Evidence for positive correlation of sensory perceptual error for forms spaced less than $1^{\circ}$ apart in the fovea was found.

If a single letter can be perceived with $80 \%$ accuracy in a $10 \mathrm{msec}$ exposure, how much must the exposure duration be increased for two, three, or four letters all to be perceived correctly on $80 \%$ of the trials? Sperling (1963) has concluded from his data that for up to four letters the visual perceptual system scans letters at the approximate rate of one every $10 \mathrm{msec}$. Thus for every additional $10 \mathrm{msec}$ of exposure one additional letter can be processed or perceived up to a limit of about four.

Approaching the problem from a different methodology, Eriksen (1966) and Eriksen and Lappin (1967) have obtained evidence indicating parallel or essentially simultaneous processing for four forms or less. When two letters were presented simultaneously at brief duration the probability that both letters would be perceived was the combinatorial probability of a single letter being perceived, i.e., $\mathrm{P}^{2}$ (Eriksen, 1966). This statistical combination of independent probabilities also described the errors, or conversely, the correct identifications, for up to four forms simultaneously presented (Eriksen \& Lappin, 1967). Earlier Schlosberg (1948) had shown that the perception of one to four simultaneously presented dots is described in terms of statistical independence.

A concept of perceptual independence has been proposed to describe these findings (Eriksen, 1966).
At any moment in time the varying sensitivities, or alternatively, error factors, in the visual perceptual system are uncorrelated for elements associated with foveal areas separated by some minimal distance, and further, there is a lack of interaction between forms simultaneously presented on these separate foveal areas. Some minimal separation on the fovea is necessary for zero correlation of error components to exist and for there to be a lack of interaction between adjacent forms. On logical grounds immediately adjacent neural elements may be expected to more likely share the same micro-environment and therefore have a positive covariance in sensitivity. Indeed, Eriksen, Munsinger, and Greenspon (1966) found evidence of positive correlation of perceptual error for forms spaced $1 / 2^{\circ}$ of visual angle apart in the fovea. The perceptual independence found by Eriksen (1966) and Eriksen and Lappin (1967) was obtained when the separate forms were spaced $1^{\circ}$ or more apart.

If the percent of trials on which all of $n$ simultaneously presented forms can be correctly identified from a single exposure is predicted by $\mathrm{p}^{\mathrm{n}}$, where $P$ is the probability of a single form being correctly identified, this would imply a parallel or essentially simultaneous processing of the separate forms. A serial scanning process that required a finite period of time to process each of the separate letters or forms would require a level of performance appreciably below that predicted by the independence model. Not only would performance depend upon the probability of a particular form being available through the noise or error in the sensory channel for processing by the scanning mechanism, but a constant time increment would have to be added for each letter scanned or processed.

The present experiment had two principle purposes. The first was to determine the increments in exposure duration needed to achieve the criterion of all presented forms identified correctly on $80 \%$ of the trials as the number of presented forms varied from one to four. The second purpose was to study the correlation of sensory-perceptual error components as forms were spaced at varying angular separations in the fovea. Prior research on the span of visual attention or apprehension has largely ignored the foveal spacing variable although it would be impossible to determine perceptual processing time for 
each letter or form unless the correlation of sensoryperceptual error between different letter locations was known.

In the present experiment, durations were determined for each $\mathrm{S}$ that yielded $80 \%$ identification accuracy when single letter displays were presented. Then the increment in exposure duration necessary for a correct identification of all letters on a display on $80 \%$ of the trials was determined for two, three, and four letter displays. In view of the evidence that perceptual independence breaks down when stimuli are spaced much closer than $1^{\circ}$ apart in the fovea, the present experiment systematically varied the effect of different foveal spacing of the form stimuli in the display. Spacings of $1 / 2,3 / 4$, and $1^{\circ}$ of angle were studied.

\section{Subjects}

\section{METHOD}

Eight undergraduate students at the University of Illinois (four males) served as paid Ss. All had normal or corrected to normal vision.

\section{Apparafus and Stimuli}

A Scientific Prototype Model GA three-field tachistoscope was used. Only two fields of the three-field tachistoscope were employed. The stimulus display was preceded and followed by dark exposure fields. The stimuli were the five capital letters $A, H, O, T$, and V, obtained from Paratype No. 11316. These letters were black mounted on white plastic cards and subtended $.2^{\circ}$ of visual angle. Field illumination was $.2 \mathrm{~mL}$ and contrast of the letters with the ground was approximately $96 \%$. The two variables studied were number of letters in a display and the spacings of these letters around a central fixation point. The display sizes ranged from one to four letters with no letter occurring more than once in a particular display. The letters were located within the corners of an imaginary square centered on the fixation point. The size of the imaginary square varied for the foveal spacings such that the outer edge of the letters would be $1 / 2,3 / 4$, or $1^{\circ}$ of angle from the center of the fixation point. Thus there were 12 different conditions in all, four display sizes at each of three foveal spacings. Twenty different stimuli were made for each of these 12 conditions.

For the single letter displays each of the five letters occurred once in each of the four positions on the squares to make 20 for each foveal distance, but for the other three display sizes, 20 stimulus patterns were selected systematically from all the various possible combinations of letters and positions. Each letter appeared in each position and with each other letter an equal number of times. All positions and combinations of positions were occupied an equal number of times for all sets of stimuli.

\section{Procedure}

All Ss were practiced before beginning the experiment. Each $\mathrm{S}$ was initially run for two or more sessions to establish approximate exposure durations for $80 \%$ accuracy in form identification. An $80 \%$ criterion was chosen for two reasons. First, it provides a sensitive measure of any processing time variable. If a $100 \%$ criterion was chosen there is always some uncertainty as to whether stimulus energy could not be reduced and Ss still maintain the $100 \%$ criterion. Second, other work in our laboratory has shown essentially linear relations between percent correct identifications of these letter forms and exposure duration in $\mathrm{msec}$ over the range of $10 \%$ better than chance to $90 \%$ correct identifications. Thus in conceptualizing the results one can translate from a percent correct variable to an exposure duration variable without having to transform the essential functional relationships.

Following the practice sessions the experimental sessions presented one, two, three, and four form displays at the $1 / 2^{\circ}$ foveal spacing and the exposure duration necessary for identification of all presented forms in their correct locations in the display on $80 \%$ of the trials was determined. In subsequent experimental sessions the four display sizes at the $3 / 4$ and $1^{\circ}$ spacing were presented. In these sessions the exposure duration for each display size was, for each $\mathrm{S}$, the duration established for $80 \%$ accuracy with the $1 / 2^{\circ}$ spacing. The order in which the four display sizes were presented during the experimental sessions was counterbalanced over Ss to prevent bias due to task habituation. Each $\mathrm{S}$ received 80 trials at each of the four display sizes and at each of the three degrees of foveal spacing.

On each trial an $\mathrm{S}$ was instructed to fixate a backlighted "X" fixation point in the center of an otherwise dark field and, following a signal from $E$, to trigger the stimulus onset when the fixation " $\mathrm{X}$ " appeared sharp and clear. His response consisted of naming the letters in a specified counterclockwise order and of saying "blank" when he came to a position which he thought to be unoccupied. A response was scored as incorrect if any of the letters or their positions were incorrectly named.

\section{RESULTS}

The exposure durations necessary to achieve the $80 \%$ accuracy criterion for one, two, three, and four form displays at the $1 / 2^{\circ}$ foveal spacing were analyzed first. These mean exposure durations are shown in Table 1. Since it might be anticipated that there was some variation around the $80 \%$ accuracy criterion, the mean percentage of trials where all forms were correctly identified is also shown in Table 1 for the four display sizes.

A two-way analysis of variance (display size and 
Table 1. Mean Exposure Duration for Correct Identification of all Presented Forms on the Designated Percentage of Trials for $12^{\circ}$ Foveal Spacing

\begin{tabular}{lllll} 
& \multicolumn{5}{c}{ Display Size } \\
\hline & 1 & 2 & 3 & 4 \\
\hline Mean Exposure Duration & 14.5 & 18.7 & 19.4 & 19.4 \\
Percent Correct & 76 & 81 & 79 & 80 \\
\hline
\end{tabular}

Ss) was performed on the duration time data of Table 1. The effects due to display sizes and to Ss were both significant beyond the .01 level $(F=27.93$, of $=3 / 21$, and $F=26.31, d f=7 / 21$, respectively). As can be seen from Table 1 the significant effect for display sizes was due to the difference between the duration required for $80 \%$ accuracy on one form displays and the larger displays. The durations necessary to identify all of the forms on two, three, and four form displays on $80 \%$ of the trials were virtually identical, all being within $.7 \mathrm{msec}$ of each other. As can also be seen, the failure to obtain duration time differences for displays of two and larger cannotbe attributed to a lower accuracy on these larger size displays. The percent of trials on which complete accuracy was achieved for all display sizes is in all cases within a few percentage points of $80 \%(F=.51, d f=3 / 21)$.

The next analysis concermed the identification accuracy for two to four form displays at the $1 / 2$, $3 / 4$, and $1^{\circ}$ foveal spacing. At these foveal spacings the different display sizes had been presented at an exposure duration that would have resulted in all presented forms being identified on $80 \%$ of the trials had they all been spaced at $1 / 2^{\circ}$ of angle (mean exposure duration $18.7 \mathrm{msec}$ ). A three-way analysis of variance (display size, foveal spacing, and $\mathrm{Ss}$ ) was employed. The effect due to foveal spacing was significant beyond the .01 level $(F=373.8$, $d f=2 / 14)$. The $S$ effect did not reach significance, indicating that the exposure duration adjustments fairly well matched Ss on overall identification accuracy. Also the main effect of display size was nonsignificant, but there was a signiflcant interaction between foveal spacing and display size $(F=5.6, d f=4 / 28, p<.01$ ). The foveal spacing by $S$ interaction was not significant, but the display size by $S$ interaction was significant beyond the .01 level, Indicating that accuracy of different Ss dropped at a different rate as the number of presented forms increased.

The interaction between foveal spacing and display size is of major interest and can be examined in Fig. 1. Here the percent of trials on which all presented forms are correctly identified is shown as a function of the number of forms presented and the foveal spacing. Figure 1 also shows for both the $3 / 4$ and $1^{0}$ foreal spacings the functions that would have been generated if perceptual independence obtained. The nature of the display size by foveal spacing interaction can be understood by comparing the empiri- cal curves with their theoretical independence curves. These latter were generated in the following way. If the error or sensitivity was uncorrelated at any moment in time between the elements in the visual system represented by the foveal locations of the separate forms, then the percent of trials on which two, three, and four forms were correctly identified would have a simple mathematical relationship to one another. The percentage for two forms would equal $x^{2}$, for three forms $x^{3}$, and for four forms $x^{4}$, where $x$ is the percent of trials where a one form display is correctly identified. Using the empirical values for two, three, and four form displays and averaging the three estimates, the value of $x$ for the $3 / 4^{\circ}$ spacing is $84 \%$ and for the $1^{\circ}$ spacing $76 \% .^{2}$ The theoretical independence curve is then generated for the two foveal spacings by: $P_{n}=x^{n}$ where $P_{n}$ is the predicted percent of trials on which all of the $n$ presented forms are identified.

For the $1^{0}$ spacing there is very good agreement between the empirical and the theoretical independence functions. This suggests that if forms are spaced $1^{\circ}$ of angle or more apart around the center of the fovea, error factors are uncorrelated, a conclusion which agrees with previous findings (Eriksen, 1966; Eriksen \& Lappin, 1965, 1967). The empirical curve for $3 / 4^{\circ}$ spacing deviates appreciably from the independence curve and in a direction that indicates a positive correlation in the sensory-perceptual processing of the separate forms in the display.

\section{DISCUSSION}

In considering input-output information processing of human Ss, it has become increasingly necessary

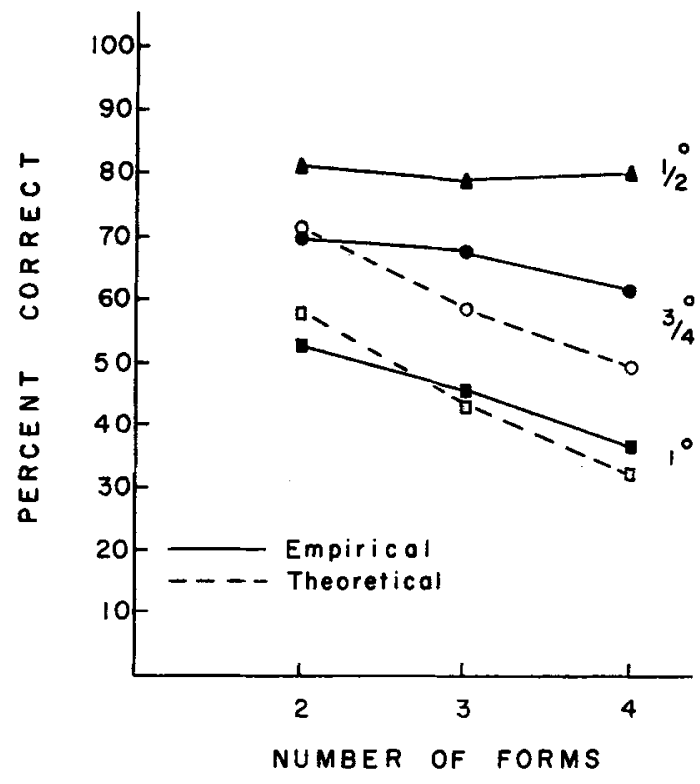

Fig. 1. Percent of trials on which all presented forms are correctly identified as a function of display size (numbers of forms) and foveal spacing. 
to break the concept of perception down into several sub-processes or stages. Garner, Hake, and Eriksen (1956) have pointed out the necessity of separating response variables from the concept of perception, and recently Broadbent (1958), Sperling (1963), and Averbach and Coriell (1961) have further reduced perception to sensory, scanning, and brief storage sub-processes. While these sub-stages are hypothetical, their conceptual value and precision are capable of assessment through appropriate converging experimental operations. From experimental data so far obtained, it would seem desirable to consider human information processing in terms of these four substages: sensory channels, brief sensory storage, scanning or noting processes, and response variables. Questions concerning whether visual information is parallel or serially processed would seem to require discussion in terms of these sub-stages, since obtained measures of capacity for simultaneous processing could be limited by characteristics of any one or more. Thus competition or interference among responses could limit the obtained results as could limitations on the brief storage capacity. Similarly, the scanning or noting process may set limits depending upon whether it is serial or multi-channel in nature. If serial, the scan time or period between scans becomes an important determiner of the obtained outcome and, if multi-channel in nature, the number of channels sets limits on simultaneous processing.

Research has typically focused upon the scanning or noting process and on the brief storage capacity (Sperling, 1963; Mackworth, 1962). Largely ignored have been the effects of the sensory channels, their associated noise or varying sensitivity, and whether independent information channels exist within a single sense modality. Results of the present experiment are quite clear in demonstrating that any attempt to determine whether multiple simultaneously presented forms are parallel or serially processed cannot ignore the foveal spacing of the separate forms and the associated problem of correlated vs uncorrelated error components. Any conclusions concerning the speed or nature of a central scanning mechanism that does not address these sensory channel considerations is highly suspect.

In order for a central scanning mechanism or noting process to operate upon information, it is first necessary that this information arrive at some central station in an adequate enough form to be so processed. It is here that the effect of the retinal locus of the form stimulation becomes important. It is possible that with too close spacing between simultaneously presented forms there is an interaction of the forms such that transmission through the sensory channel is impaired or distorted. Backward and forward masking phenomena might be relevant in this consideration (Raab, 1963). With brief visual presentations where the single form identification hit rate is less than
1.00 , a major problem is the conceptualization of the joint probability of two or more simultaneously presented forms being available to the scanner. Completely different conclusions concerning the serial vs parallel processing character of the central scanning mechanism would be obtained depending upon whether a correlation of $1.00, .50$, or .00 is assumed between the accuracy of the sensory processing of each of the separate simultaneously stimulating forms.

The present data suggest that if forms are spaced at least $1^{\circ}$ apart around the central foveal fixation, the obtained results are quite well described by assuming that the sensory-perceptual error in processing each of the forms is independent or uncorrelated. With closer foveal spacings the results no longer are consistent with an assumption of independence. Rather, at $3 / 4^{\circ}$ spacing the optained results are consistent with an interpretation of positively correlated processing error.

The duration time data obtained for the $1 / 2^{\circ}$ foveal spacing necessary to achieve the $80 \%$ criterion for one to four form displays can be interpreted in terms of a still higher degree of positive correlation of processing error. Due to a linear relation between exposure duration and percent correct over the accuracy range investigated, the negatively accelerated function for the $1 / 2^{\circ}$ spacing, which asymptotes at two form displays, can be compared with the negatively accelerated functions obtained for the $3 / 4$ and $1^{\circ}$ spacing. The much more rapidly approached asymptote for the $1 / 2^{\circ}$ spacing is consistent with an interpretation in terms of an increasing degree of positive correlation among error components as forms are spaced closer together within the fovea.

It is tempting to ascribe the error components completely to the sensory channel. However, such a conclusion would be premature. Given an $80 \%$ identification hit rate for single form presentations, one could ascribe trial to trial variation in accuracy as due to noise in the sensory channel which results in incomplete information being made available to the noting or scanning process on some trials. This would assume that if the information were available from the sensory channel the scanning mechanism is error free. An alternative interpretation would be that there is noise or error in both the sensory channel and in the scanning process, and the observed hit rate of $80 \%$ is the joint function of error in the sensory channel and error in the scanning mechanism. Such a formulation would lead to the question as to whether the sensory system error and the scanning system error were independent. Finally, although difficult to defend, an assumption could be made that all the error resides in the scanning mechanism and that there is no sensory system error. ${ }^{3}$

Irrespective of how the error is conceptualized, the data obtained on the increment in exposure duration necessary to maintain the $80 \%$ accuracy criterion 
as display sizes increase from one to four forms is difficult to reconcile with a serial processing mechanism. It certainly is inconsistent with Sperling's (1963) conclusion that forms are scanned at the rate of one every $10 \mathrm{msec}$ up to approximately four forms. When the simultaneously presented forms were spaced only $1 / 2^{\circ}$ of angle apart in the fovea only a $4.2 \mathrm{msec}$ increase in exposure duration was required for two form displays to be perceived at the same accuracy level as one form displays. And essentially no further increment in exposure duration was required for three and four form displays to achieve the same accuracy criterion.

This obtained function between exposure duration and display size is what would be expected with an essentially simultaneous processing of up to four forms and a high positive correlation of the error components for the different form locations. In fact if we attribute the error solely to the sensory channels, a high positive correlation among the error components should yield a very sensitive test of serial processing. If the correlation were perfect, then on any given trial all presented forms would be available for processing or none of them would be. Under these circumstances a linear increase in exposure duration with an increase in display size would be obtained with the rate of the increase, $t$, reflecting the scanning time per letter or the period of the scanner. As the correlation of the error components was reduced from 1.00 to .00 the increment in exposure duration as each new letter was added to the display would increase even more. Not only would the duration have to be increased by time $t$ to process an additional letter, but also an additional increment in exposure duration would be necessary to insure that all presented forms were available for scanning or processing.

Given an $80 \%$ identification accuracy for single form displays and complete independence of the forms in the sensory channels, the probability that two simultaneously presented forms would be available to the scanner from the sensory channel on a given trial is .64. Exposure duration necessary to maintain the $80 \%$ criterion would not only have to be increased by time $t$ for the scanner to process the additional letter but also by an additional amount necessary to raise the probability that both forms would be available to the scanner on $80 \%$ of the trials. In the present data, even at the $1^{\circ}$ foveal spacing there is no suggestion that to maintain an $80 \%$ accuracy cri- terion, exposure duration would need to be increased any more than was necessary to insure that all presented forms were available to the scanner on $80 \%$ of the trials.

\section{References}

Averbach, E., \& Coriell, A. S. Short-term memory in vision. Bell Sys. tech. J., 1961, 40, 309-328.

Broadbent, D. E. Perception and communication. New York: Pergamon Press, 1958.

Eriksen, C. W. Independence of successive inputs and uncorrelated error in visual form perception. $J$. exp. Psychol., 1966, 72, 26-35.

Eriksen, C. W., \& Lappin, J. S. Internal perceptual system noise and redundancy in simultaneous inputs in form identification. Psychon. Sci., 1965, 2, 351-352.

Eriksen, C. W., \& Lappin, J. S. Independence in the perception of simultaneously presented forms at brief durations. J. exp. Psychol., 1967, 73, 468-472.

Eriksen, C. W., Munsinger, H. L., \& Greenspon, T. S. Identification versus same-different judgment: An interpretation in terms of uncorrelated perceptual error. J. exp. Psychol., 1966, 72, 20-25.

Garner, W. R., Hake, H. W., \& Eriksen, C. W. Operationism and the concept of perception. Psychol. Rev., 1956, 63, 149-159.

Mackworth, J. F. The visual image and the memory trace. Canad, $J$. Psychol., 1962, 16, 55-59.

Raab, D. H. Backward masking. Psychol. Bull., 1963, 60, 118-129. Schlosberg, H. A probability formulation of the Hunter-Sigler effect. J. exp. Psychol., 1948, 38, 155-167.

Sperling, G. A model for visual memory tasks. Hum. Factors, 1963 , $5,19-31$.

\section{Notes}

1. This investigation was supported by Public Health Service Research Grant MH-1206 and a Public Health Service Research Career Program Award K6-MH-22,014.

2. Due to a decrease in acuity as forms are spaced further from the center of the fovea, identification accuracy for one form displays would be expected to be less at $1^{\circ}$ spacing than at $34^{\circ}$. Identification of one form displays was not obtained for the $3 / 4$ and $1^{\circ}$ spacing at the $18.7 \mathrm{msec}$ exposure but the loss in acuity can be seen by comparing the one form identification accuracy at the $14.5 \mathrm{msec}$ duration at $1 / 2,3 / 4$, and $1^{\circ}$. The respective percentages are 76,74 , and 70. Also the estimate of the single form identification accuracy will be biased to the extent that non-independence obtains. Positive correlation will lead to a systematic overestimate for the single form accuracy. However, this bias works against the effect observed in Fig. 1.

3. Theoretically, it is also possible that the noting process or scanner decreases in efficiency when the displayed information increases. Thus the identification accuracy for any given form would be less in a multiform display than if only single forms were presented. This possibility can be discounted since Eriksen and Lappin (1967) found that the percent identification accuracy for any given form remained essentially invariant as the number of presented forms increased.

(Accepted for publication April 18, 1967.) 